\section{THE ONLINE CONCUSSION AWARENESS TRAINING TOOL}

Shelina Babul, lan Pike, Kate Turcotte. BC Injury Research and Prevention Unit, Canada; University of British Columbia, Canada

\subsection{6/injuryprev-2016-042156.262}

Background The online Concussion Awareness Training Tool (CATT-www.cattonline.com) includes three toolkits providing training in the recognition and treatment of concussion: Medical Professionals-MP; Parents, Players and Coaches-PPC; School Professionals-SP.

Objective To create an accessible, regularly updated online resource based upon the Zurich Consensus Statement on Concussion in Sport and evidence-based resources, intended to support standardise concussion diagnosis, treatment and management. Each toolkit includes a self-paced learning module as well as tailored resources.

CATT MP aims to standardise practice in a clinical setting with a focus on the paediatric patient.

CATT PPC speaks to concussion identification and management, with Smartphone accessible resources: Concussion Response Tool and Questions to Ask Your Doctor.

CATT SP includes Return-to-Learn protocol and resources for teachers, counsellors and others in the school setting.

Results For each of the CATT toolkits, evaluation was undertaken with a pre-post intervention survey design. Sample sizes of at least 33 were required to compare the change in scores with the power to detect a large effect size of 0.5 .

CATT MP was launched mid April, 2013. Physicians demonstrated significant positive change in concussion practices $(\mathrm{p}=0.001)$, and significant change in knowledge by those treating more than 10 concussions/yr $(\mathrm{p}=0.039)$. Nurses had significant positive change in practices $(\mathrm{p}=0.005)$ and attitudes $(\mathrm{p}=0.035)$. CATT PPC was launched mid June, 2014. Parents demonstrated significant positive change in concussion knowledge $(\mathrm{p}=0.002)$. CATT SP is anticipated for launch in November 2015, with an accompanying evaluation.

Conclusions Concussion is an under-diagnosed medical condition, requiring both physical and mental rest. CATT addresses this gap by increasing knowledge and awareness among appropriate audiences. Good concussion management can reduce related health problems and the risk of long-term brain damage.

\section{HEADS UP FOR YOUTH SPORT CONCUSSION: MOVING FROM AWARENESS TO EVALUATION OF BEHAVIOURAL INTENTION}

Kelly Sarmiento, Robin Lee, Ann Dellinger, Grant Baldwin. Centres for Disease Control and Prevention/National Centre for Injury Prevention and Control, USA

\subsection{6/injuryprev-2016-042156.263}

Background Children and adolescents account for an estimated $65 \%$ of emergency department visits for sports and recreationrelated traumatic brain injuries, including concussions. Immediate identification and appropriate response to a concussion can help reduce the risk of short- or long-term health problems that can affect thinking, learning, behaviour, and/or emotions.

Description of the Problem Combining innovative and evidencebased communication strategies, CDC created HEADS UP.
HEADS UP is a series of educational initiatives grounded in audience preferences for content, design, format, and distribution channels. The content is specifically built to draw attention to what was considered an under-reported and under-identified public health problem and is designed to improve awareness, early identification and management of sports-related concussion. Results In the last decade, CDC's HEADS UP has partnered with over 85 organisations, received over 200 million media impressions, distributed more than 6 million print resources, and obtained over 40 million social-media impressions. In addition, through HEADS UP, CDC has trained over 3 million sports coaches and health care professionals through online training courses required by many state policies and sports programs. Evaluation results indicate improvements in knowledge and attitudes towards concussion after exposure to HEADS UP materials.

Conclusion CDC's HEADS UP demonstrates how a health communication initiative can play a critical role in driving the science of an important health issue and can help support implementation of policies on a large-scale. As CDC embarks on the next stage of HEADS UP, continued emphasis will be placed on adapting to the changing landscape of concussion research and awareness and identifying effective approaches for improving both behaviours and the culture of concussion nationwide.

\section{PARENT AND PLAYER CONCUSSION KNOWLEDGE AND FACILITATORS OF APPROPRIATE MANAGEMENT IN YOUTH ICE HOCKEY}

${ }^{1}$ Amanda M Black, ${ }^{2}$ Shelina Babul, ${ }^{3,4}$ Alberto Nettel-Aguire, ${ }^{1,3,4}$ Carolyn Emery. ${ }^{1}$ Sport Injury Prevention Research Centre, Faculty of Kinesiology, University of Calgary, Canada; ${ }^{2}$ Department of Paediatrics/Pathology and Laboratory Medicine, University of British Columbia, Canada; ${ }^{3}$ Alberta Children's Hospital Research Institute, Canada; ${ }^{4}$ Departments of Paediatrics and Community Health Sciences, Cumming School of Medicine, University of Calgary, Canada

\subsection{6/injuryprev-2016-042156.264}

Background Concussions are a significant burden in youth ice hockey that can have serious health consequences if not identified and managed appropriately. Parents' role in facilitating identification and appropriate concussion management as well as child education is vital. This project aims to understand parent and player concussion knowledge and potential barriers to physician diagnosis and appropriate return to play after concussion.

Methods Using a mixed methods study design, 63 players (ages 11-14) and 82 parents from Vancouver and Calgary (Canada) completed a survey on concussion knowledge. Qualitative interviews of 6 mothers and 7 fathers explored the barriers and facilitators associated with physician follow-up after a concussion.

Results All parents and $93.6 \%$ players identified concussion as a brain injury. When asked to identify concussion symptoms from a list with 8 concussion and 8 distractor symptoms, parents and players had similar mean scores (12.7 and $12.3 / 16$ respectively). Only $50.8 \%$ of parents and $14.3 \%$ of players were able to identify the correct course of action for all three scenario-based concussion management questions. Finally, only 59.8\% of parents and $11.1 \%$ of players recognised the term "graduated return to play protocol". A preliminary analysis of themes from the interviews highlighted barriers to physician follow-up: Parents' belief that the concussion was not serious, concussion symptoms 
resolving prior to physician visit, and physician availability. Facilitators of appropriate behaviour: Team personnel explaining to the parents the importance of physician visits at time of injury and a team protocol that enforces physician-based management.

Conclusions Concussion awareness is improving but there are still gaps in hockey parents' and players' understanding of management. Understanding beliefs, barriers and facilitators of proper concussion management behaviour may assist with reducing the consequences that can arise due to mismanagement.

\section{Drowning and Water Safety}

\section{Parallel Tue 1.6}

\section{POOL FENCING BYLAWS AND DROWNING RATES AMONG CHILDREN}

Tessa Clemens, Michael Rotondi, Hala Tamim, Alison Macpherson. Faculty of Kinesiology and Health Science, York University, Canada

\subsection{6/injuryprev-2016-042156.265}

Background The majority of fatal drownings in Canada occur in natural bodies of water; however private backyard pools are consistently the most common setting where children under 5 years of age drown. A number of studies conducted primarily in Australia and the United States have provided evidence that pool fencing reduces the risk of drowning among children. No long term analysis of pool fencing and municipal bylaws as a factor affecting the risk of childhood drowning in Canada has been published.

Methods Using a multi-level ecologic study design, the drowning death rate in Ontario municipalities with isolation fence and gate legislation was compared to that in municipalities with less or no legislation. Individual level descriptive analysis was conducted using data collected from files at the Ontario Provincial Coroner's office for all children under the age of 5 who suffered drowning deaths in private backyard pools over a fifteen year period. Drowning death rates were calculated per 100000 population. Denominators for rates were yearly estimates of population under the age of 5 for each municipality. Poisson regression methods were used to estimate relative risks and 95\% confidence intervals. Additionally, univariate analysis was conducted and descriptive statistics were reported to summarise the characteristics of childhood backyard pool drownings.

Results During the study period, 54 children under the age of 5 drowned in a private backyard pool in Ontario. The highest death rate was found among 2 year olds $(0.9$ per 100 000) and the male to female ratio was $3.5: 1$. The majority of children $(61 \%)$ drowned after accessing an unobstructed pool directly from the residence. (Results of Poisson regression to be updated). Conclusions The results of the study demonstrate the need for isolation fencing bylaws that do not allow direct access from the building, and do not allow fences to be "grandfathered" under existing legislation.

\section{SWIMMING ABILITY AND DROWNING PREVENTION - DO THEY HAVE SOMETHING IN COMMON? A NORDIC CASE STUDY}

${ }^{1}$ Riitta Vienola, ${ }^{2}$ Hafthor B Gudmundsson, ${ }^{3}$ Kristiina Heinonen. ${ }^{1}$ Arcada, University of Applied Sciences, Finland; ${ }^{2}$ Iceland University of Education, Iceland; ${ }^{3}$ Finnish Swimming Teaching and Lifesaving Federation

\subsection{6/injuryprev-2016-042156.266}

Background In 1996 the Nordic Countries defined the term swimming ability. A person can be said to be able to swim when he, after being immersed in water, can swim continuously for 200 metres, of which at least $50 \mathrm{~m}$ on backstroke. Since then the countries have been collecting data concerning swimming ability statistics. Drownings are one of the leading causes of death worldwide (WHO 2015). The first poster concerning the topic was presented in the World Conference on Drowning Prevention in Potsdam in 2013. The updated data will be presented in 2016 aiming to find correlation between swimming ability and drowning rates (1996-2016) within the Nordic Countries.

Methods Each country has been collecting their own data. There is variation in data collecting methods. Data has been collected in different years (2011-2013) depending on country-specific policies. A table has been created to clarify the findings.

Results The highest rates in swimming ability came from Iceland, $95 \%$ of the children and $96 \%$ of the adults can swim and the lowest rates came from Norway, 50\%, no data available for adults. (Sweden 92\%, no data for adults; Denmark 79/66\%; Finland $72 / 68 \%)$. The highest drowning rates came from Finland, 2.3/100,000 and the lowest from Iceland 0.62 (Denmark 1.3, Norway 1.03, Sweden 0.84). There are some indications of a correlation between swimming ability and low drowning rates.

Conclusions The swimming ability of a nation seems to play an important role for drowning prevention but there are other important factors (e.g. alcohol abuse, cultural differences, falling through the ice) not related to swimming ability. However, these other factors related to drownings do not decrease the importance of swimming ability, vice versa. The co-operation within the Nordic Countries is special and needs to be emphasised more. While the rest of the world is having difficulties in defining swimming ability and self-rescue skills, a closer look at the Nordic numbers is recommended.

\section{TWENTY YEARS OF PFD NON-WEARING AND WEARING AMONG CHILD AND YOUTH BOATING IMMERSION VICTIMS IN CANADA}

1,2Peter Barss, ${ }^{1}$ Karlyn Olsen, ${ }^{1} J a n e$ Hamilton, 'Shelley Dalke. 'Canadian Red Cross Water Safety Program; ${ }^{2}$ Division of Occupational and Environmental Health, School of Population and Public Health, University of British Columbia, Vancouver, Canada

\subsection{6/injuryprev-2016-042156.267}

Background Boating is the most frequent activity for waterrelated immersion deaths in Canada. Central in immersion/ drowning is non-wearing of personal flotation devices (PFDs). Although most boating victims are adult males, wearing and compliance with regulations was assessed for children 0-14-years-old and youth 15-19 victims of immersion deaths. Indigenous were compared with other ethnicities.

Methods Annual Red Cross collection of 1991-2010 Canadian coroner data by structured questionnaire. Analysis included activity, purpose, personal, equipment, environment factors. 